\title{
The Strong Law of Large Numbers for Weighted Averages Under Dependence Assumptions
}

\author{
Tapas K. Chandra ${ }^{1}$ and Subhashis Ghosal ${ }^{1}$
}

Received February 21, 1995; revised December 7, 1995

Strong laws of large numbers (SLLN) for weighted averages are proved under various dependence assumptions when the variables are not necessarily independent or identically distributed. The results considerably extend the existing results. Weighted versions of the Marcinkiewicz-Zygmund SLLN are also formulated and proved under a similar set up. It seems that such results are not known even for independent and identically distributed random variables.

KEY WORDS: Asymptotically quadrant sub-independence; MarcinkiewiczZygmund strong laws; maximal inequalities; mixing conditions; mixingale difference; strong laws; weighted averages.

\section{INTRODUCTION}

Let $\left\{X_{n}\right\}$ be a sequence of random variables (rvs) and $\left\{a_{n}\right\}$ be a sequence of positive weights; put $A_{n}=\sum_{k=1}^{n} a_{k}$ and $T_{n}=A_{n}^{-1} \sum_{k=1}^{n} a_{k}\left(X_{k}-E X_{k}\right)$. Using a result of Chandra and Ghosal ${ }^{(3)}$ stated as Theorem 2.1, we establish the almost sure convergence of $\left\{T_{n}\right\}$ to zero where $\left\{X_{n}\right\}$ is AQSI or $\varphi$-mixing or *-mixing. (For the definition of AQSI, see Section 2.) Our results extend considerably those of Jamison et al., ${ }^{(7)}$ Pruitt, ${ }^{(9)}$ Rohatgi, ${ }^{(10)}$ and Etemadi. ${ }^{(5)}$

We also consider the analogues of Marcinkiewicz-Zygmund strong law of large numbers (MZSLLN in short) for weighted averages under very general conditions; it appears that similar results are not known even under the i.i.d. setup.

All the results are stated in Section 2 and the proofs are given in Section 3.

\footnotetext{
'Division of Theoretical Statistics and Mathematics, Indian Statistical Institute, 203 B. T. Road, Calcutta 700035, India.
} 


\section{MAIN RESULTS}

Here we shall assume that the weight sequence $\left\{a_{n}\right\}$ satisfies the following conditions:

(a) $A_{n} \rightarrow \infty$ and $a_{n} / A_{n} \rightarrow 0$.

(b) For all $j \geqslant 1,\langle j\rangle:=\left\{k \geqslant 1: j-1<A_{k} / a_{k} \leqslant j\right\} \subset\{1, \ldots, K j\}$ for some positive integer $K$.

Remark 2.1. Assumption (b) is slightly stronger that the assumption that

$$
\#\left\{k \geqslant 1: A_{k} / a_{k} \leqslant j\right\} \leqslant K j, \quad \forall j \geqslant 1
$$

made in Jamison et al. ${ }^{(7)}$ It will be clear from the proofs that Eq. (2.1) suffices in case the rvs are stochastically dominated by a rv $X$ with $E|X|<\infty$ (with $E|X|^{p}<\infty$ for MZSLLN). Finally, (b) is satisfied in the unweighted case and more generally, whenever $\left\{A_{n} / a_{n}\right\}$ is increasing and Eq. (2.1) holds.

Definition 2.1. A sequence $\left\{X_{n}\right\}$ of rvs is said to be asymptotically quadrant sub-independent (AQSI) if there exists a nonnegative sequence $\{q(m)\}$ such that $q(m) \rightarrow 0$ and $\forall i \neq j$,

$$
\begin{aligned}
& P\left\{X_{i}>s, X_{j}>t\right\}-P\left\{X_{i}>s\right\} P\left\{X_{i}>t\right\} \leqslant q(|i-j|) \alpha_{i j}(s, t), \quad s, t>0, \\
& P\left\{X_{i}<s, X_{j}<t\right\}-P\left\{X_{i}<s\right\} P\left\{X_{i}<t\right\} \leqslant q(|i-j|) \beta_{i j}(s, t), \quad s, t<0
\end{aligned}
$$

where $\alpha_{i j}(s, t)$ and $\beta_{i j}(s, t)$ are nonnegative numbers.

This dependence condition is a useful weakening of the definition of AQI proposed by Birkel. ${ }^{(1)}$ The AQSI condition is satisfied by many mixing sequences (see Birkel $^{(1)}$ ) as well as by pairwise $m$-dependent and pairwise negative quadrant dependent sequences.

We shall use the following result stated in Chandra and Ghosal. ${ }^{(3)}$

Theorem 2.1. Let $\left\{X_{n}\right\}$ be a square integrable AQSI sequence satisfying

$$
\sup _{i \neq j} \int_{0}^{\infty} \int_{0}^{\infty} \alpha_{i j}(s, t) d s d t<\infty, \quad \sup _{i \neq j} \int_{-\infty}^{0} \int_{-\infty}^{0} \beta_{i j}(s, t) d s d t<\infty
$$

Assume that

$$
\begin{aligned}
\text { (i) } & \sup _{n \geqslant 1}\left(\sum_{k=1}^{n} E\left|X_{k}\right| / f(n)\right)<\infty, \\
\text { (ii) } & \sum_{j=1}^{\infty}(f(j))^{-2} \operatorname{var}\left(X_{j}\right)<\infty, \\
\text { (iii) } & \sum_{m=1}^{\infty} q(m) \sum_{j=m+1}^{\infty}(f(j))^{-2}<\infty
\end{aligned}
$$


where $\{f(n)\}$ is a positive sequence increasing to infinity. Then

$$
(f(n))^{-1} \sum_{k=1}^{n}\left(X_{k}-E X_{k}\right) \rightarrow 0 \quad \text { a.s. }
$$

We shall replace the condition of identical distribution by considering the functions

$$
G(y)=\sup _{n \geqslant 1} n^{-1} \sum_{k=1}^{n} P\left\{\left|X_{k}\right|>y\right\}
$$

and

$$
\hat{G}(y)=\sup _{n \geqslant 1} A_{n}^{-1} \sum_{k=1}^{n} a_{k} P\left\{\left|X_{k}\right|>y\right\}
$$

For various dependence conditions, the SLLN for the weighted averages will be proved under the assumptions

$$
\begin{aligned}
& \int_{0}^{\infty} G(y) d y<\infty \\
& \int_{0}^{\infty} \hat{G}(y) d y<\infty
\end{aligned}
$$

and

$$
\sum_{n=1}^{\infty} P\left\{\left|X_{n}\right|>A_{n} / a_{n}\right\}<\infty
$$

It is easily observed that a single sufficient condition for Eqs. (2.6)-(2.8) is

$$
\int_{0}^{\infty} G^{*}(y) d y<\infty
$$

where

$$
G^{*}(y)=\sup _{n \geqslant i} P\left\{\left|X_{n}\right|>y\right\}
$$

Condition Eq. (2.9) is equivalent to saying that $\left\{X_{n}\right\}$ is stochastically dominated by an integrable random variable. Finally, in the unweighted case, $\hat{G}(y)=G(y)$.

Theorem 2.2. Let $\left\{X_{n}\right\}$ be an AQSI sequence satisfying

$$
\begin{aligned}
& \sup _{i \neq j} a_{i} a_{j} \int_{0}^{A_{j} / a_{j}} \int_{0}^{A_{i} / a_{i}} \alpha_{i j}(s, t) d s d t<\infty \\
& \sup _{i \neq j} a_{i} a_{j} \int_{-A_{j} / a_{j}}^{0} \int_{-A_{i} / a_{i}}^{0} \beta_{i j}(s, t) d s d t<\infty
\end{aligned}
$$


and $\sum_{m=1}^{\infty} q(m) \sum_{j=m+1}^{\infty} A_{j}^{-2}<\infty$. If Eqs. (2.6)-(2.8) hold, then

$$
A_{n}^{-1} \sum_{k=1}^{n} a_{k}\left(X_{k}-E X_{k}\right) \rightarrow 0 \quad \text { a.s. }
$$

The SLLN given by Eq. (2.12) also holds, as shown later, under the $\varphi$-mixing and $*$-mixing conditions. (In this context, we follow the terminologies of McLeish ${ }^{(8)}$ and Hall and Heyde ${ }^{(6)}$ respectively.)

Theorem 2.3. Suppose $\left\{X_{n}\right\}$ is a $\varphi$-mixing sequence with mixing coefficients $\left\{\varphi_{m}\right\}$ of size -1 . If Eqs. (2.6)-(2.8) are satisfied, then Eq. (2.12) holds.

Theorem 2.4. If $\left\{X_{n}\right\}$ is *-mixing and Eqs. (2.6)-(2.8) are satisfied, then Eq. (2.12) holds.

We shall now consider the weighted versions of the MZSLLN under various dependence conditions, which can be, roughly speaking, described as follows:

Let $\left\{X_{n}\right\}$ satisfy suitable dependence conditions and let

$$
\tilde{G}(y)=\sup _{n \geqslant 1}\left(\sum_{k=1}^{n} a_{k}^{1 / p}\right)^{-1} \sum_{k=1}^{n} a_{k}^{1 / p} P\left\{\left|X_{k}\right|>y\right\}
$$

Assume that

$$
\begin{aligned}
& \int_{0}^{\infty} y^{p-1} G(y) d y<\infty \\
& \int_{0}^{\infty} y^{p-1} \tilde{G}(y) d y<\infty
\end{aligned}
$$

and

$$
\sum_{n=1}^{\infty} P\left\{\left|X_{n}\right|^{p}>A_{n} / a_{n}\right\}<\infty
$$

Then

$$
A_{n}^{-1 / p} \sum_{k=1}^{n} a_{k}^{1 / p} X_{k} \rightarrow 0 \quad \text { a.s. }
$$


if either $0<p<1$ or $1 \leqslant p<2$ and $E X_{n}=0 \forall n \geqslant 1$. (Note that $\tilde{G}(y)=G(y)$ in the unweighted case and $\vec{G}(y)=\hat{G}(y)$ if $p=1$. A single sufficient condition for Eqs. (2.14) $-(2.16)$ is

$$
\int_{0}^{\infty} y^{p-1} G^{*}(y) d y<\infty
$$

where $G^{*}(y)$ is as defined in Eq. (2.10). This is the same as saying that $\left\{X_{n}\right\}$ is stochastically bounded by a random variable $X$ with $E|X|^{p}<\infty$.)

It is well known that in the unweighted setup, the case $0<p<1$ is trivial and the MZSLLN holds irrespective of any dependence condition. Lemma 3.2 of Section 3 shows that the same is true in the weighted case also.

From now on, we shall assume that $1 \leqslant p<2$ and $E X_{n}=0 \forall n \geqslant 1$.

Definition 2.2. A sequence $\left\{X_{n}\right\}$ of rvs is called asymptotically almost negatively associated (AANA) if there is a nonnegative sequence $q(m) \rightarrow 0$ such that for all $m, k \geqslant 1$,

$$
\begin{aligned}
& \operatorname{cov}\left(f\left(X_{m}\right), g\left(X_{m+1}, \ldots, X_{m+k}\right)\right) \\
& \quad \leqslant q(m)\left(\operatorname{var}\left(f\left(X_{m}\right)\right) \operatorname{var}\left(g\left(X_{m+1}, \ldots, X_{m+k}\right)\right)\right)^{1 / 2}
\end{aligned}
$$

for every coordinatewise increasing continuous function $f$ and $g$ so that the right-hand side of Eq. (2.19) is finite.

The family of AANA sequences contain negatively associated (in particular independent) sequences (with $q(m)=0 \quad \forall m \geqslant 1$ ). The condition roughly means that asymptotically the future is almost negatively associated with the present. For an example of AANA which is not negatively associated, consider $X_{n}=Y_{n}+\alpha_{n} Y_{n+1}$ where $Y_{1}, Y_{2}, \ldots$ are i.i.d $N(0,1)$ and $\alpha_{n} \rightarrow 0, \alpha_{n}>0$.

Theorem 2.5. Assume that $\left\{X_{n}\right\}$ is AANA with $\sum_{m=1}^{\infty} q^{2}(m)<\infty$. If Eqs. (2.14)-(2.16) are satisfied then Eq. (2.17) holds.

The following version of the MZSLLN can be proved for $\varphi$-mixing sequences.

Theorem 2.6. Assume that $\left\{X_{n}\right\}$ is $\varphi$-mixing with $\sum_{m=1}^{\infty} \varphi^{1 / 2}(m)<$ $\infty$. Then Eqs. (2.14)-(2.16) imply Eq. (2.17).

The crux of the proofs depends on a maximal inequality applied on the truncated variables. A version of the MZSLLN for AQSI sequences is also stated later. However, this conclusion is not as sharp as those in 
Theorems 2.5 and 2.6 because the maximal inequality available here is not so powerful.

Let

$$
v(n)=\inf \left\{k \geqslant 1: A_{k} \geqslant 2^{\prime \prime}\right\}
$$

Since $A_{k} \rightarrow \infty, v(n)<\infty$. Also $a_{k} / A_{k} \rightarrow 0$, so without loss of generality, assume that $a_{k} / A_{k}<1 / 2$. Then it easily follows that $2^{n} \leqslant A_{v(n)}<2^{n+1}$; consequently $\{v(n)\}$ is a subsequence of integers.

Theorem 2.7. Assume that $\left\{X_{n}\right\}$ is AQSI with $\sum_{m=1}^{\infty} q(m)<\infty$ and for all $i \neq j$,

$$
\begin{gathered}
a_{i}^{1 / p} a_{j}^{1 / p} \int_{0}^{\left(A_{j} / a_{j}\right)^{1 / p}} \int_{0}^{\left(A_{i} / a_{i}\right)^{1 / p}} \alpha_{i j}(s, t) d s d t \leqslant D\left(1+E Y_{i}^{2}+E Y_{j}^{2}\right) \\
a_{i}^{1 / p} a_{j}^{1 / p} \int_{-\left(A_{j} / a_{j}\right)^{1 / p}}^{0} \int_{-\left(A_{i} / a_{i}\right)^{1 / p}}^{0} \beta_{i j}(s, t) d s d t \leqslant D\left(1+E Y_{i}^{2}+E Y_{j}^{2}\right)
\end{gathered}
$$

where $Y_{n}=a_{n}^{1 / p} X_{n} I\left\{\left|X_{n}\right|^{p} \leqslant A_{n} / a_{n}\right\}+A_{n}^{1 / p} I\left\{X_{n}>\left(A_{n} / a_{n}\right)^{1 / p}\right\}-A_{n}^{1 / p} I\left\{X_{n}<\right.$ $\left.-\left(A_{n} / a_{n}\right)^{1 / p}\right\}$ and $D$ is a constant. If Eqs. (2.14)-(2.16) hold, then

$$
A_{n}^{-1 / p}(\log n)^{-1} \sum_{k=1}^{n} a_{k}^{1 / p} X_{k} \rightarrow 0 \quad \text { a.s. }
$$

provided $\log v(n+1)=O(\log v(n))$.

Remark 2.2. The hypotheses on $\alpha_{i j}(s, t)$ and $\beta_{i j}(s, t)$ may seem to be rather awkward. However, it is satisfied if $\sum_{m=1}^{\infty} q(m)<\infty$ and

$$
\begin{array}{ll}
P\left\{X_{i}>s, X_{j}>t\right\} \leqslant(1+q(|i-j|)) P\left\{X_{i}>s\right\} P\left\{X_{j}>t\right\}, & s, t>0 \\
P\left\{X_{i}<s, X_{j}<t\right\} \leqslant(1+q(|i-j|)) P\left\{X_{i}<s\right\} P\left\{X_{j}<t\right\}, & s, t<0
\end{array}
$$

Relation Eq. (2.22) is much weaker than demanding that $\left\{X_{n}\right\}$ is *-mixing with coefficients $\{q(m)\}$.

\section{PROOFS}

For the proofs of Theorems 2.2-2.4, we shall use the following result. Throughout this section, $C$ will stand for a generic constant.

Lemma 3.1. Let $\left\{X_{n}\right\}$ be any stochastic sequence such that Eqs. (2.6) $-(2.8)$ hold. Then 
(a) $\sum_{k=1}^{\infty} A_{k}^{-2} a_{k}^{2} E\left(X_{k}^{2} I\left\{\left|X_{k}\right| \leqslant A_{k} / a_{k}\right\}\right)<\infty$,

(b) $A_{n}^{-1} \sum_{k=1}^{n} a_{k} E\left(\left|X_{k}\right| I\left\{\left|X_{k}\right|>A_{k} / a_{k}\right\}\right) \rightarrow 0$,

(c) $\sup _{n \geqslant 1} A_{n}^{-1} \sum_{k=1}^{n} a_{k} E\left|X_{k}\right|<\infty$

Proof. The expression in (a) is dominated by

$$
\begin{aligned}
& \sum_{j=1}^{\infty} \sum_{k \in\langle j\rangle}(j-1)^{-2} E\left(X_{k}^{2} I\left\{\left|X_{k}\right| \leqslant j\right\}\right) \\
& \leqslant C \sum_{j=1}^{\infty} \sum_{k \in\langle j\rangle} \sum_{l=j}^{\infty} l^{-3} \sum_{n=1}^{j} \int_{n-1}^{n} y P\left\{\left|X_{k}\right|>y\right\} d y \\
& \leqslant C \sum_{l=1}^{\infty} l^{-3} \sum_{n=1}^{l} \int_{n-1}^{n} y\left(\sum_{j=1}^{l} \sum_{k \in\langle j\rangle} P\left\{\left|X_{k}\right|>y\right\}\right) d y
\end{aligned}
$$

Since for $j \neq j^{\prime},\langle j\rangle \cap\left\langle j^{\prime}\right\rangle=\varnothing$ and $\langle j\rangle \subset\{1, \ldots, K j\}$, the right-hand side of Eq. (3.1) is smaller than

$$
\begin{aligned}
C \sum_{l=1}^{\infty} l^{-3} \sum_{n=1}^{l} \int_{n-1}^{n} y K l G(y) d y & \leqslant C \sum_{n=1}^{\infty} n^{-1} \int_{n-1}^{n} y G(y) d y \\
& \leqslant C \int_{0}^{\infty} G(y) d y
\end{aligned}
$$

To prove (b), fix $N \geqslant 1$ and let $n>N$. Then the expression in (b) is equal to

$$
\begin{aligned}
& A_{n}^{-1} \sum_{k=1}^{N} a_{k} \int_{A_{k} / a_{k}}^{\infty} P\left\{\left|X_{k}\right|>y\right\} d y+A_{n}^{-1} \sum_{k=N+1}^{\infty} a_{k} \int_{A_{k} / a_{k}}^{\infty} P\left\{\left|X_{k}\right|>y\right\} d y \\
& \quad+A_{n}^{-1} \sum_{k=1}^{n} A_{k} P\left\{\left|X_{k}\right|>A_{k} / a_{k}\right\} d y
\end{aligned}
$$

The first term in Eq. (3.3) is less than

$$
A_{n}^{-1} N\left(\max _{1 \leqslant k \leqslant N} a_{k}\right) \int_{0}^{\infty} G(y) d y
$$

which goes to zero as $n \rightarrow \infty$ for any fixed $N$. The third term goes to zero by Kronecker's lemma. For the second term, let $M$ be a given positive integer and $N$ is so chosen that $A_{k} / a_{k}>M \forall k>N$. Thus the second term is dominated by

$$
A_{n}^{-1} \sum_{k=1}^{n} a_{k} \int_{M}^{\infty} P\left\{\left|X_{k}\right|>y\right\} d y \leqslant \int_{M}^{\infty} \hat{G}(y) d y
$$


Since $M$ can be arbitrarily large, (b) is proved. The proof of (c) is elementary.

Proof of Theorem 2.2. Put $Y_{n}=a_{n} X_{n} I\left\{\left|X_{n}\right| \leqslant A_{n} / a_{n}\right\}+A_{n} I\left\{X_{n}>\right.$ $\left.A_{n} / a_{n}\right\}-A_{n} I\left\{X_{n}<-A_{n} / a_{n}\right\}$. In view of Lemma 3.1(b) and Eq. (2.8), it suffices to show that

$$
A_{n}^{-1} \sum_{k=1}^{n}\left(Y_{k}-E Y_{k}\right) \rightarrow 0 \quad \text { a.s. }
$$

Note that $\left\{Y_{n}\right\}$ also form an AQSI sequence, i.e., $\forall i \neq j$,

$$
\begin{array}{ll}
P\left\{Y_{i}>s, Y_{j}>t\right\}-P\left\{Y_{i}>s\right\} P\left(Y_{j}>t\right\} \leqslant q(|i-j|) \alpha_{i j}^{*}(s, t), & s, t>0 \\
P\left\{Y_{i}<s, Y_{j}<t\right\}-P\left\{Y_{i}<s\right\} P\left\{Y_{j}<t\right\} \leqslant q(|i-j|) \beta_{i j}^{*}(s, t), & s, t<0
\end{array}
$$

where

$$
\alpha_{i j}^{*}(s, t)= \begin{cases}\alpha_{i j}\left(s / a_{i}, t / a_{j}\right), & \text { if } 0<s<A_{i}, \quad 0<t<A_{j}, \\ 0, & \text { otherwise }\end{cases}
$$

and

$$
\beta_{i j}^{*}(s, t)= \begin{cases}\beta_{i j}\left(s / a_{i}, t / a_{j}\right), & \text { if }-A_{i}<s<0, \quad-A_{j}<t<0, \\ 0, & \text { otherwise }\end{cases}
$$

In view of Lemma $3.1(\mathrm{a})$ and (c), $\left\{Y_{n}\right\}$ satisfies the hypotheses of Theorem 2.1 with $f(n)=A_{n}$, and so Eq. (3.6) follows.

Proof of Theorem 2.3. Without loss of generality, assume that $E X_{n}=0$ $\forall n \geqslant 1$. Set $Y_{n}=a_{n} X_{n} / A_{n}$. In view of Lemma 3.1(b) and Kronecker's lemma, it suffices to show that

$$
\sum_{n=1}^{\infty}\left(Y_{n}-E\left(Y_{n} I\left\{\left|Y_{n}\right| \leqslant 1\right\}\right)\right) \quad \text { converges a.s. }
$$

By taking $g_{n}(x)=\min \left(x^{2}, 1\right), d_{n}=1$ and $r=2$, it follows from Lemma 2.9 of $\mathrm{McLeish}^{(8)}$ that we need to verify only

$$
\sum_{n=1}^{\infty}\left(A_{n}^{-2} a_{n}^{2} E\left(X_{n}^{2} I\left\{\left|X_{n}\right| \leqslant A_{n} / a_{n}\right\}+P\left\{\left|X_{n}\right|>A_{n} / a_{n}\right\}\right)<\infty\right.
$$

In view of Lemma 3.1(a) and Eq. (2.8), the proof is now complete. 
Proof of Theorem 2.4. Let $Y_{n}=a_{n} X_{n} I\left\{\left|X_{n}\right| \leqslant A_{n} / a_{n}\right\}$. By Lemma 3.1(a) and (c), the hypotheses of Theorem 2.20 of Hall and Heyde ${ }^{(6)}$ are satisfied for the sequence $Y_{n}-E Y_{n}, n \geqslant 1$, with $b_{n}=A_{n}$. Consequently, $A_{n}^{-1} \sum_{k=1}^{n}\left(Y_{k}-E Y_{k}\right) \rightarrow 0$ a.s. which leads to Eq. (2.12).

The key idea of proving Theorem 2.5 is to establish the result first for a suitable subsequence and use a maximal inequality (see Theorem A.l of Appendix) to lift it to the whole sequence. The following analogue of Lemma 3.1 will be exploited.

Lemma 3.2. Let $\left\{X_{n}\right\}$ be any sequence of rvs satisfying Eqs. (2.14)-(2.16). Then

(a) $\sum_{k=1}^{\infty} A_{k}^{-2 / p} a_{k}^{2 / p} E\left(X_{k}^{2} I\left\{\left|X_{k}\right|^{p} \leqslant A_{k} / a_{k}\right\}\right)<\infty$,

(b) $\sum_{k=1}^{\infty} A_{k}^{-1 / p} a_{k}^{1 / p} E\left(\left|X_{k}\right| I\left\{\left|X_{k}\right|^{p} \leqslant A_{k} / a_{k}\right\}\right)<\infty$ if $0<p<1$, $A_{n}^{-1 / p} \sum_{k=1}^{n} a_{k}^{1 / p} E\left(\left|X_{k}\right| I\left\{\left|X_{k}\right|>A_{k} / a_{k}\right\}\right) \rightarrow 0$ if $1 \leqslant p<2$,

(c) $\sum_{n=1}^{\infty} A_{v(n)}^{-2 / p} \sum_{k=1}^{v(n+1)} a_{k}^{2 / p} E\left(X_{k}^{2} I\left\{\left|X_{k}\right|^{p} \leqslant A_{k} / a_{k}\right\}\right)<\infty$,

(d) $\left.\sum_{n=1}^{\infty} A_{v(n)}^{-2 / p} \sum_{k=1}^{v(n+1)} A_{k}^{2 / p} P\left\{\left|X_{k}\right|^{p}>A_{k} / a_{k}\right\}\right)<\infty$

(Here $v(n)$ is as defined in Eq. (2.20).)

Proof. Proof of part (a) is similar to that of part (a) of Lemma 3.1. Part (b) for $0<p<1$ can also be, proved analogously. To establish part (b) for $1 \leqslant p<2$, split the expression in three parts as done in Eq. (3.3); we then need to verify

$$
\lim _{N \rightarrow \infty} \limsup _{n \rightarrow \infty} A_{n}^{-1 / p} \sum_{k=N+1}^{n} a_{k}^{1 / p} E\left(\left|X_{k}\right| I\left\{\left|X_{k}\right|^{p}>A_{k} / a_{k}\right\}\right)=0
$$

Given a positive integer $M$, find $N \geqslant 1$ such that $A_{k} / a_{k}>M \forall k>N$. Thus

$$
\begin{aligned}
& A_{n}^{-1 / p} \sum_{k=N+1}^{n} a_{k}^{1 / p} E\left(\left|X_{k}\right| I\left\{\left|X_{k}\right|^{p}>A_{k} / a_{k}\right\}\right) \\
& \leqslant A_{n}^{-1 / p} \sum_{j=M+1}^{\infty} \sum_{k \leqslant n: A_{k} / a_{k} \leqslant j} a_{k}^{1 / p} \int_{(j-1)^{1 / p}}^{j^{1 / p}} P\left\{\left|X_{k}\right|>y\right\} d y \\
& \leqslant A_{n}^{-1 / p} \sum_{j=M+1}^{\infty} \int_{(j-1)^{1 / p}}^{j^{1 / p}}\left(\sum_{k=1}^{m} a^{1 / p} P\left\{\left|X_{k}\right|>y\right\}\right) d y
\end{aligned}
$$


where $m=\min (n, K j)$. Using the fact that

$$
\sum_{k=1}^{m} a_{k}^{1 / p} \leqslant m^{1-1 / p}\left(\sum_{k=1}^{m} a_{k}\right)^{1 / p} \leqslant(K j)^{1-1 / p} A_{n}^{1 / p}
$$

the right-hand side of Eq. (3.10) is dominated by

$$
C \sum_{j=M+1}^{\infty} j^{1-1 / p} \int_{(j-1)^{1 / p}}^{j^{1 / p}} \tilde{G}(y) d y \leqslant C \int_{M}^{\infty} y^{p-1} \widetilde{G}(y) d y
$$

which goes to zero as $M \rightarrow \infty$ (i.e., as $N \rightarrow \infty$ ).

To prove part (c), we write the expression as

$$
\begin{aligned}
& \sum_{k=1}^{\infty} a_{k}^{2 / p} E\left(X_{k}^{2} I\left\{\left|X_{k}\right|^{p} \leqslant A_{k} / a_{k}\right\}\right) \sum_{n: v(n+1) \geqslant k} A_{v(n)}^{-2 / p} \\
& \quad \leqslant \sum_{k=1}^{\infty} a_{k}^{2 / p} E\left(X_{k}^{2} I\left\{\left|X_{k}\right|^{p} \leqslant A_{k} / a_{k}\right\}\right) \sum_{n: v(n+1) \geqslant k} 2^{-2 n / p}
\end{aligned}
$$

Let $n_{k}=\inf \{n \geqslant 1: v(n) \geqslant k\}$. Then

$$
\sum_{n:(n+1) \geqslant k} 2^{-2 n / p} \leqslant C 2^{-2 n_{k} / p} \leqslant C A_{v(n k)}^{-2 / p} \leqslant C A_{k}^{-2 / p}
$$

and so (c) follows from part (a). Part (d) is proved similarly.

Proof of Theorem 2.5. It suffices to prove that

$$
A_{n}^{-1 / p} \sum_{k=1}^{n}\left(Y_{k}-E Y_{k}\right) \rightarrow 0 \quad \text { a.s. }
$$

where $Y_{n}=a_{n}^{1 / p} X_{n} I\left\{\left|X_{n}\right|^{p} \leqslant A_{n} / a_{n}\right\}+A_{n}^{1 / p} I\left\{X_{n}>\left(A_{n} / a_{n}\right)^{1 / p}\right\}-A_{n}^{1 / p} I\left\{X_{n}<\right.$ $\left.-\left(A_{n} / a_{n}\right)^{1 / p}\right\}$. Put $U_{n}=Y_{n}-E Y_{n}$ and $T_{n}=\sum_{k=1}^{n} U_{k}$. By the proof of Theorem 1 of Chandra and Ghosal, ${ }^{(4)} E T_{n}^{2} \leqslant C \sum_{k=1}^{n} E U_{k}^{2}$; thus Lemma 3.2(c) and (d) implies that

$$
A_{v(n)}^{-1 / p} \sum_{k=1}^{n(n)} U_{k} \rightarrow 0 \quad \text { a.s. }
$$

For $m \geqslant 1$, let $n=n_{m}$, be such that $v(n) \leqslant m<v(n+1)$. In view of Eq. (3.16), it now suffices to show that

$$
Z_{m}:=A_{m}^{-1 / p} \sum_{k=v(n)+1}^{v(n+1)} U_{k} \rightarrow 0 \quad \text { a.s. }
$$


Fix $\varepsilon>0$. We have

$$
\begin{aligned}
\sum_{n=1}^{\infty} P & \left\{\max \left(\left|Z_{m}\right|: v(n)<m<v(n+1)\right)>\varepsilon\right\} \\
\leqslant & \sum_{n=1}^{\infty} P\left\{\max _{v(n)<m<v(n+1)}\left|\sum_{k=v(n)+1}^{v(n+1)} U_{k}\right|>\varepsilon A_{v(n)}^{1 / p}\right\} \\
\leqslant & C \sum_{n=1}^{\infty} A_{v(n)}^{-2 / p} \sum_{k=1}^{v(n+1)}\left(a _ { k } ^ { 2 / p } E \left(X_{k}^{2} I\left\{\left|X_{k}\right|^{p} \leqslant A_{k} / a_{k}\right\}\right.\right. \\
& \left.+A_{k}^{2 / p} P\left\{\left|X_{k}\right|^{p}>A_{k} / a_{k}\right\}\right)
\end{aligned}
$$

above, we have used the maximal inequality given in Theorem A.l of Appendix. The right-hand side of Eq. (3.18) is finite by Lemma 3.1(c) and (d); thus Eq. (3.17) follows.

Proof of Theorem 2.6. Let $Y_{n}=a_{n}^{1 / p} X_{n} I\left\{\left|X_{n}\right|^{p} \leqslant A_{n} / a_{n}\right\}$. As $\left\{\varphi_{n}\right\}$ is trivially of size -1 , Theorem 2.7 of McLeish ${ }^{(8)}$ implies that $U_{n}:=Y_{n}-E Y_{n}$ is a mixingale (difference) sequence with $\psi_{m}=\varphi_{m}^{1 / 2}$. Also $\forall i<j$,

$$
E\left(U_{i} U_{j}\right)=E\left(U_{i} E\left(U_{j} \mid X_{1}, \ldots, X_{i}\right)\right) \leqslant \psi_{j-i}\left\|U_{i}\right\|_{2}\left\|U_{i}\right\|_{2}
$$

Using straightforward arguments (see, e.g., Lemma 1 of Chandra ${ }^{(2)}$ ) we obtain $A_{v(n)}^{-1 / p} \sum_{k=1}^{v(n)} a_{k}^{1 / p} U_{k} \rightarrow 0$ a.s. The rest of the proof can be completed as in Theorem 2.5 with the aid of McLeish's ${ }^{(8)}$ maximal inequality.

Proof of Theorem 2.7. It is easy to see that $\operatorname{var}\left(\sum_{k=1}^{n} Y_{k}\right) \leqslant$ $C \sum_{k=1}^{n}\left(1+E Y_{k}^{2}\right)$, and so $A_{v(n)}^{-1 / p} \sum_{k=1}^{n} a_{k}^{1 / p}\left(Y_{k}-E Y_{k}\right) \rightarrow 0$ a.s. It now remains to show that $\forall \varepsilon>0$,

$$
\sum_{n=1}^{\infty} P\left\{\max \left(\left|Z_{n}\right|: v(n)<m<v(n+1)\right)>\varepsilon\right\}<\infty
$$

where $Z_{m}=A_{m}^{-1 / p}(\log m)^{-1} \sum_{k=y_{m}\left(n_{m}\right)+1}^{w\left(n_{m}+1\right)}\left(Y_{k}-E Y_{k}\right)$ and $n_{m}$ is as in the proof of Theorem 2.5. By Theorem A.2 of Appendix,

$$
\begin{aligned}
& P\left\{\max \left(\left|Z_{m}\right|: v(n)<m<v(n+1)\right)>\varepsilon\right\} \\
& \quad \leqslant P\left\{\max \left(\left|\sum_{k=v(n)+1}^{m}\left(Y_{k}-E Y_{k}\right)\right|>\varepsilon A_{v(n)}^{1 / p} \log v(n)\right\}\right. \\
& \left.\quad \leqslant C A_{v(n)}^{-2 / p}(\log v(n))\right)^{-2} \times\left(\frac{\log v(n+1)}{\log 3}+2\right)^{2} \sum_{k=v(n)+1}^{v(n+1)}\left(1+E Y_{k}^{2}\right)
\end{aligned}
$$

Hence Eq. (3.20) follows immediately from Eq. (3.21) and Lemma 3.2. 
Remark 3.1. A close examination of the proof of Theorem 2.7 yields the following variation of it:

Assume that all the hypotheses of Theorem 2.7 are satisfied except that Eqs. (2.14) and (2.16) are strengthened to

$$
\int_{0}^{\infty} y^{p-1}(\log y)^{2} G(y) d y<\infty
$$

and

$$
\sum_{n=1}^{\infty}(\log n)^{2} P\left\{\left|X_{n}\right|^{p}>A_{n} / a_{n}\right\}<\infty
$$

respectively. Then

$$
A_{n}^{-1 / p} \sum_{k=1}^{n} a_{k}^{1 / p} X_{k} \rightarrow 0 \quad \text { a.s. }
$$

If actually $\left\{X_{n}\right\}$ are pairwise independent, then the strengthening Eq. (3.23) is not necessary since here we can use the truncation $Y_{n}=a_{n} X_{n}$ $I\left\{\left|X_{n}\right|^{p} \leqslant A_{n} / a_{n}\right\}$.

The SLLN problem for weighted sums of pairwise independent and identically distributed rvs was studied extensively by Rosalsky. ${ }^{(11)}$ However, Rosalsky's ${ }^{(11)}$ results and the current ones do not include each other.

\section{APPENDIX A}

Theorem A.1. Let $X_{1}, \ldots, X_{n}$ be mean zero, square integrable rvs such that Eq. (2.19) holds for $1 \leqslant m<k+m \leqslant n$ and for all coordinatewise increasing continuous functions $f$ and $g$ whenever the right side of Eq. (2.19) is finite. Let $A^{2}=\sum_{m=1}^{n-1} q^{2}(m)$ and $\sigma_{k}^{2}=E X_{k}^{2}, k \geqslant 1$. Then

$$
P\left\{\max _{1 \leqslant k \leqslant n}\left|\sum_{i=1}^{k} X_{i}\right| \geqslant \varepsilon\right\} \leqslant 2 \varepsilon^{-2}\left(A+\left(1+A^{2}\right)^{1 / 2}\right)^{2} \sum_{k=1}^{n} \sigma_{k}^{2}
$$

For a proof, see Chandra and Ghosal, ${ }^{(4)}$ (Theorem 1).

Theorem A.2. Let $X_{1}, \ldots, X_{n}$ be square integrable rvs such that there exist numbers $a_{1}^{2}, \ldots, a_{n}^{2}$ satisfying $E\left(X_{m+1}+\cdots+X_{m+p}\right)^{2} \leqslant a_{m+1}^{2}+\cdots+a_{m+p}^{2}$, $\forall m, p$. Then we have $E\left(\max _{1 \leqslant k \leqslant n}\left(\sum_{i=1}^{k} X_{i}\right)^{2}\right) \leqslant((\log n / \log 3)+2)^{2} \sum_{i=1}^{\prime \prime} a_{i}^{2}$.

The result is an extension of the well known Rademacher-Mensov inequality. A proof can be found in Chandra and Ghosal, ${ }^{(3)}$ (Theorem 10). 


\section{REFERENCES}

1. Birkel, T. (1992). Laws of large numbers under dependence assumptions. Statist. Prob. Lett. 14, 355-362.

2. Chandra, T. K. (1991). Extensions of Rajchman's strong law of large numbers. Sankhyā, Ser. A 53, 118-121.

3. Chandra, T. K., and Ghosal, S. (1993). Some elementary strong laws of large numbers: a review. Technical Report \#22/93, Indian Statistical Institute.

4. Chandra, T. K., and Ghosal, S. (1996). Extensions of the strong law of large numbers of Marcinkiewicz and Zygmund. Acta Marh. Hung. 72(3) (to appear).

5. Etemadi, N. (1983). Stability of sums of weighted random variables. J. Multivariate Anal. 13, 361-365.

6. Hall, P., and Heyde, C. C. (1980). Martingale Limit Theory and Its Application. Academic Press, New York.

7. Jamison, B., Orey, S., and Pruitt, W. E. (1965). Convergence of weighted averages of independent random variables. Z. Wahrsch. Verw. Gebiele 4, 40-44.

8. McLeish, D. L. (1975). A maximal inequality and dependent strong laws. Anm. Prob. 3. 829-839.

9. Pruitt. W. E. (1966). Summability of independent random variables. J. Math. Mech. 15, 769-776.

10. Rothatgi, V. K. (1971). Convergence of weighted sums of independent random variables. Proc. Cambridge Phil. Soc. 69, 305-307.

11. Rosalsky, A. (1987). Strong stability of normed sums of pairwise i.i.d. random variables. Bull. Inst. Math. Acad. Sinica 15, 203-219. 